\title{
Sensores y equipos de captación automática de datos en los trabajos prácticos de Física y Química de Secundaria y Bachillerato: el uso de Arduino
}

\author{
María Susana Alegre Buj \\ Diputación General de Aragón. Departamento de Educación, Cultura y Deporte. Profesores de Educación \\ Secundaria, FP y RR.EE. Aragón. España.amsalegreb@educa.aragon.es \\ María José Cuetos Revuelta (iD \\ Universidad Internacional de La Rioja. Facultad de Educación. Área de Didáctica de las Matemáticas \\ y de las Ciencias Experimentales.La Rioja.España.mjose.cuetos@unir.net
}

[Recibido: 9 junio 2020. Revisado: 3 septiembre 2020. Aceptado: 20 septiembre 2020]

\begin{abstract}
Resumen: Los estudiantes nacidos en una era digital requieren metodologías ajustadas a la sociedad del siglo XXI, donde la competencia digital se hace indispensable. Al mismo tiempo, se debe construir un ambiente de trabajo que estimule el aprendizaje de manera más efectiva a través de las interacciones entre los alumnos y las tecnologías de la información y la comunicación (TIC). En este estudio se recoge el diseño de una batería de prácticas de Física y Química por medio de sensores y equipos de captación automática de datos mediante el uso de la plataforma libre Arduino, que sean fáciles de implementar en diferentes niveles educativos. Se describen en detalle las actividades planteadas, los circuitos montados y el código empleado. Se pretende de esta manera fomentar la enseñanza-aprendizaje de estas asignaturas y dar un enfoque contextualizado a temas abstractos pero que los alumnos puedan encontrar en su vida diaria (como los cambios en la materia, el concepto de onda y la energía). Arduino se puede utilizar para exponer a los alumnos conceptos complejos, haciendo que sean más comprensibles y significativos para los estudiantes y consiguiendo que los alumnos estén más motivados a través de la tecnología y el trabajo colaborativo.
\end{abstract}

Palabras clave: Arduino; Trabajos prácticos; Física y Química; Secundaria y Bachillerato; Motivación.

Use of sensors and automatic data collection equipment in the practical work of Physics and Chemistry of middle and high school: the Arduino platform

Abstract: Students born in a digital age require methodologies adjusted to the society of the 21 st century, where digital competence is essential. At the same time, a work environment must be built to stimulate learning more effectively through interactions between students and information and communication technologies (ICT). This study includes the design of a battery of Physics and Chemistry practices by means of sensors and automatic data capture equipment using the free Arduino platform, which are easy to implement at different educational levels. The activities planned, the circuits set up and the code used are described in detail. In this way, it is intended to promote the teaching-learning of these subjects, give a contextualized approach to abstract topics but that students can find in their daily lives (such as changes in matter, wave concept and energy). Arduino can be used to expose students to complex concepts, making them more understandable and meaningful to students, and making students more motivated through technology and collaborative work.

Keywords: Arduino platform; Practical activities; Physics and Chemistry; Middle and High School; Motivation

Para citar este artículo: Alegre Buj M. S., Cuetos Revuelta M. J. (2021) Sensores y equipos de captación automática de datos en los trabajos prácticos de Física y Química de Secundaria y Bachillerato: el uso de Arduino. Revista Eureka sobre Enseñanza y Divulgación de las Ciencias 18(1), $1202 . \quad$ doi: 10.25267/Rev_Eureka_ensen_divulg_cienc.2021.v18.i1.1202

\section{Introducción}

Los resultados del informe PISA 2018 para España (la prueba de la OCDE que mide las competencias de los alumnos de 15 años en ciencias, matemáticas y comprensión lectora)

\author{
Revista Eureka sobre Enseñanza y Divulgación de las Ciencias \\ Universidad de Cádir. APAC-Eureka. ISSN: 1697-011X \\ bttp:/ / doi.org/10.25267/Rev_Eureka_ensen_divulg_cienc.2021.v18.i1.1202 \\ bttp:/ / reuredc.uca.es
}


muestran los peores resultados en ciencias de estos años, recomendando la realización de actividades no memorísticas que creen conceptos e ideas científicas reales y que ayuden a los estudiantes a establecer una conexión entre actividades prácticas, ideas científicas y los problemas de la vida real.

Para ello, los docentes deberían diseñar unas prácticas de laboratorio bien estructuradas y con los recursos adecuados. La idea es que tanto los profesores como los estudiantes puedan realizar experimentos prácticos y simples con el uso de dispositivos portátiles y fáciles de manejar. Al mismo tiempo, los estudiantes nacidos en la era digital requieren metodologías ajustadas de enseñanza y aprendizaje que incorporen nuevas tecnologías como, por ejemplo, los sensores. Los sensores son elementos que se emplean para medir alguna magnitud de la Física o Química, o para detectar la presencia de alguna sustancia o material en algún sistema (Carranza et al. 2013). Por tanto, hoy en día, la integración de la tecnología basada en sensores para el aprendizaje mejora la calidad de la enseñanza de las ciencias, especialmente para una mejor calidad de mediciones (Prima et al. 2017).

Este tipo de sistemas de adquisición de datos es cada vez más utilizado en los laboratorios para prácticas educativas, sobre todo la plataforma Arduino, tanto en el nivel superior, (Oliveira y Hedengren 2019, Rodriguez-Sánchez, Torrado-Carvajal, Vaquero, Borromeo y Hernandez-Tamames 2016) como en las escuelas de secundaria, donde su uso se ha extendido cada vez más, sobre todo en el ámbito de la Física (Garrigós et al. 2017, Kinchin 2018, Moya 2018, Parreira y Yao 2018, Prima et al. 2017). En los diversos estudios se concreta que el uso de sensores y equipos de captación automática de datos ayuda a promover en los estudiantes la comprensión de los aspectos abstractos de fenómenos o conceptos que estudian, debido a que hacen predicciones, observan y comparan los resultados.

El objetivo de este trabajo es presentar esta plataforma a los profesores de Física y Química y mostrar cómo se puede utilizar para actividades diversas, con estudiantes de Secundaria y Bachillerato de todas las edades y con un bajo coste. Para su aplicación se han buscado fenómenos que habitualmente supongan una dificultad para ser entendidos por los estudiantes y que se puedan encontrar en su vida diaria. Los discentes pueden presentar dificultades a la hora de entender ciertos conceptos físicos y químicos, ya que, con los métodos de instrucción tradicional, muchos estudiantes toman pasivamente apuntes de clase y simplemente memorizan la información y las ecuaciones a aplicar, pero no analizan los conceptos subyacentes para comprender los fenómenos completamente. Entre estas dificultades se encuentran los cambios en la materia y la relación que existe entre las variables presión y temperatura (Pozo y Gómez 2009) o el concepto de onda, debido a que los estudiantes suelen asimilar a la onda desde la mecánica de la partícula (Pérez Carmona y Esper 2005). Tienden a otorgar propiedades macroscópicas a las partículas o al calor, sin comprender las teorías en dominios específicos como lo son en la Física y la Química (Blanco López y Prieto Ruz 2004) porque no asimilan bien los conceptos, y lo único que hacen es aprender memorísticamente en vez de desarrollar un pensamiento y procedimiento asociado a la educación científica (Pozo y Gómez 2009). Lo que lleva a los estudiantes a creer que los temas de ciencias están desconectados y que las ideas que les enseñan no están relacionadas con experiencias reales (Rywalt, Varney y Mutton 2019).

A pesar de que las metodologías activas ayudan a los estudiantes a establecer conexiones entre los conceptos científicos y las ecuaciones matemáticas que les enseñan en la clase (Aufschnaiter y Aufschnaiter 2007), en la mayoría de los centros aún se siguen empleando métodos de aprendizaje estrictamente tradicionales, en muchos casos porque carecen de los equipos adecuados debido a la falta de espacio, tiempo o fondos. Debido a la versatilidad de la plataforma Arduino, su bajo coste, que es de tipo open source, fácil de usar, configurar y 
mantener y funciona tanto en Windows como Linux y Mac (Jamieson 2011), se convierte en un recurso ideal para construir experimentos de ciencias utilizando componentes comunes de laboratorio (Kinchin 2018).

El objetivo principal del presente trabajo es el de mostrar una serie de actividades prácticas de Física y Química diseñadas por medio de sensores y equipos de captación automática de datos (mediante Arduino). A través de este objetivo principal se pueden extraer los siguientes objetivos específicos: primero, analizar los aportes de los sensores y equipos de captación automática de datos al proceso de enseñanza-aprendizaje de alumnos no universitarios; diseñar una batería de 6 prácticas para realizar en Física y Química; y, finalmente, introducir a los alumnos al trabajo colaborativo internándoles en el mundo tecnológico, para que entiendan cómo funcionan actualmente elementos de uso habitual.

\section{Propuesta de trabajo}

\section{Contextualización curricular}

La competencia digital se define en el actual currículum como aquella que «implica el uso creativo, crítico y seguro de las TIC para alcanzar los objetivos relacionados con el trabajo, la empleabilidad, el aprendizaje, el uso del tiempo libre, la inclusión y la participación en la sociedad» y además, «requiere de conocimientos relacionados con el lenguaje específico básico: textual, numérico, icónico, visual, gráfico y sonoro, así como sus pautas de decodificación y transferencia» (BOE 2015, p.10). Es una competencia que se aborda de forma transversal en todas las materias y abarca conocimientos y destrezas asociadas a la creación de contenidos en diferentes formatos digitales, uso seguro de la red, resolución de problemas sencillos de hardware y software y la búsqueda y manejo de la información.

La actual Ley de Educación (LOMCE 2014) incorpora la materia de Tecnologías de la Información y la Comunicación (TIC) como opcional en el bloque de asignaturas específicas del curso $4^{\circ}$ de la ESO, y como TIC I y II en $1^{\circ}$ y $2^{\circ}$ de Bachillerato, respectivamente. En $4^{\circ}$ de la ESO no se imparte programación, pero sí en Bachillerato para ayudar a solucionar problemas de la vida real. En el bloque de programación, se considera que las actividades propuestas se realicen con lenguajes de programación orientados a objetos (como ocurre con Arduino), más intuitivos y visuales, que los lenguajes de programación estructurada. Por lo que esta propuesta serviría de iniciación en Secundaria o bien se podría trabajar de forma conjunta en Bachillerato entre las asignaturas de Física y Química y TIC, estableciendo sinergias.

A pesar de que las Ciencias en general y, la Física y la Química en particular, son disciplinas proclives al trabajo y adquisición de la competencia digital, las TIC no se utilizan mucho en las clases de Ciencias y, cuando se hace, generalmente se realiza con fines expositivos y manejadas por el profesorado (Area, Cepeda y Romero 2018). Es por ello, que en estas prácticas se busca la construcción del conocimiento a través de un aprendizaje significativo, duradero y que permita a los estudiantes afrontar retos en el futuro, con la integración de las TIC como recurso didáctico central. Se pretende conseguir el desarrollo de las competencias potenciadas por la tecnología, especialmente aquellas relacionadas con la gestión del conocimiento (INTEF 2017) donde las TIC ocupan un lugar importante en nuestra sociedad, caracterizada por sus constantes cambios, complejidad y diversidad.

De este modo, las competencias que se quiere que los estudiantes alcancen con este conjunto de prácticas son:

-Competencia matemática y competencias básicas en ciencia y tecnología, necesaria para que los alumnos puedan integrarse en la sociedad de forma activa, construyendo el conocimiento mediante un aprendizaje significativo. 
-Competencia digital por el uso del Arduino y su software para gestionar, tratar, procesar y presentar la información.

-Competencia de sentido de iniciativa y espíritu emprendedor, a través de la participación de los alumnos en el desarrollo de estos proyectos donde tienen que proponer ideas y convertirlos en realidad.

-Competencia sociales y cívicas, fomentando el trabajo colaborativo.

-Competencia de aprender a aprender, propiciando que los alumnos sean protagonistas principales de su propio aprendizaje.

\section{Metodología de trabajo}

Al ser Arduino una plataforma libre, existen numerosos ejemplos de aplicaciones en Internet, sin embargo, una de las mayores dificultades encontradas a este respecto ha sido filtrar las fuentes fiables e incorporarles una sólida fundamentación científica. Tras la búsqueda, recopilación y comprobación de experiencias, finalmente se realiza un diseño total de 6 prácticas.

Las prácticas diseñadas han sido planteadas para poder aplicarlas en las asignaturas de Física y Química a alumnos de diversas edades, de Secundaria o Bachillerato, y, en general, se pueden realizar en cualquier centro educativo, ya que lo único que deben poseer son la placa de Arduino, los sensores a utilizar en cada experiencia, los componentes esenciales (conductores, resistencias, y leds) y un ordenador donde esté instalado el software de Arduino para realizar los códigos de los programas.

Del mismo modo, las actividades se pueden adaptar al nivel de los alumnos y ajustarse, en otros casos, para la adquisición de una amplia gama de conjuntos de habilidades específicas, profundizando en la comprensión de diferentes principios y conceptos abstractos de Física y Química.

Cada práctica está planificada para realizarse en 1 o 2 sesiones (de 50 minutos cada una) dependiendo de la habilidad del grupo de estudiantes. Han sido diseñadas como actividades de colaboración para trabajo en grupos de 3-4 personas. En cada actividad el profesorado puede adaptar fácilmente el modelo a su contexto educativo y promover la participación de sus estudiantes.

Se plantea cada sesión para que los alumnos aprendan haciendo y sepan cómo aplicar en su vida real los conocimientos que van adquiriendo. Esta forma de trabajar aumenta la motivación de los estudiantes y les ayuda a estructurar su conocimiento, desarrollar procesos de razonamiento e implementar habilidades de aprendizaje autodirigido (Kilroy 2004).

La metodología concreta para llevar a cabo en la batería de prácticas varía, dependiendo de la actividad experimental a realizar. Incluyendo desde experiencias perceptivas e interactivas, hasta realización de circuitos y posterior comprobación.

La idea es conseguir la construcción de un aprendizaje permanente con dos partes diferenciadas: modelo expositivo-académico inicial con la plataforma Arduino, seguida de la realización de la simulación para que los sensores capten la información y la muestren por la pantalla, el display, leds y/o buzzer.

\section{Prácticas propuestas}

A continuación, se explican en detalle cada una de las actividades diseñadas. El código empleado ha sido registrado y está disponible para el uso de cualquier investigador interesado (Alegre Buj 2020). 
En la tabla 1 se presentan los contenidos y objetivos a tratar, así como la estructura seguida en cada una de las prácticas.

Tabla 1. Resumen de las prácticas recopiladas.

\begin{tabular}{|c|c|c|c|}
\hline Práctica & Contenidos & Objetivos & Estructura \\
\hline Energía y temperatura & $\begin{array}{l}\text { Calor y energía. } \\
\text { Relación entre } \\
\text { presión y } \\
\text { temperatura }\end{array}$ & $\begin{array}{l}\text { Comprender el } \\
\text { aumento de la } \\
\text { temperatura de un gas } \\
\text { al someterlo a una } \\
\text { compresión adiabática }\end{array}$ & $\begin{array}{l}\text { Explicación del funcionamiento de } \\
\text { Arduino y del sensor } \\
\text { Realización de la práctica } \\
\text { Debate sobre las conclusiones obtenidas }\end{array}$ \\
\hline Medir distancias & $\begin{array}{l}\text { Cinemática } \\
\text { Ondas }\end{array}$ & $\begin{array}{l}\text { Comprender el } \\
\text { funcionamiento de un } \\
\text { sensor de sonido para } \\
\text { medir la distancia a la } \\
\text { que se encuentran los } \\
\text { objetos. }\end{array}$ & $\begin{array}{l}\text { Explicación del funcionamiento del } \\
\text { sensor } \\
\text { Realización de la práctica } \\
\text { Debate sobre las conclusiones obtenidas }\end{array}$ \\
\hline Mando a distancia & $\begin{array}{l}\text { Ondas } \\
\text { electromagnéticas } \\
\text { Mandos de } \\
\text { infrarrojos }\end{array}$ & $\begin{array}{l}\text { Conocer los rayos } \\
\text { infrarrojos, ondas } \\
\text { electromagnéticas y } \\
\text { cómo funcionan en los } \\
\text { mandos a distancia }\end{array}$ & $\begin{array}{l}\text { Explicación del funcionamiento del } \\
\text { sensor } \\
\text { Realización de la práctica } \\
\text { Debate sobre las conclusiones obtenidas }\end{array}$ \\
\hline $\begin{array}{l}\text { Temperatura y } \\
\text { humedad }\end{array}$ & $\begin{array}{l}\text { Conservación de } \\
\text { alimentos. }\end{array}$ & $\begin{array}{l}\text { Formular la hipótesis de } \\
\text { trabajo para la } \\
\text { resolución de un } \\
\text { problema real como } \\
\text { detonador del proceso } \\
\text { de enseñanza- } \\
\text { aprendizaje. } \\
\text { Conocer los factores } \\
\text { que influyen en la } \\
\text { conservación de los } \\
\text { alimentos. }\end{array}$ & $\begin{array}{l}\text { Lluvia de ideas sobre qué factores } \\
\text { influyen en los procesos de } \\
\text { conservación } \\
\text { Explicación del funcionamiento del } \\
\text { sensor } \\
\text { Realización de la práctica } \\
\text { Debate sobre las conclusiones obtenidas }\end{array}$ \\
\hline Vúmetro & Ondas & $\begin{array}{l}\text { Conocer el efecto que } \\
\text { produce el nivel de } \\
\text { presión sonora en la } \\
\text { salud auditiva }\end{array}$ & $\begin{array}{l}\text { Explicación del funcionamiento del } \\
\text { sensor } \\
\text { Realización de la práctica } \\
\text { Debate sobre las conclusiones obtenidas }\end{array}$ \\
\hline $\mathrm{pH}$ y notas musicales & $\mathrm{pH}$ & $\begin{array}{l}\text { Conocer la relación } \\
\text { entre el pH y la } \\
\text { conductividad y generar } \\
\text { notas musicales a través } \\
\text { de la placa de Arduino }\end{array}$ & $\begin{array}{l}\text { Explicación del funcionamiento del } \\
\text { sensor } \\
\text { Realización de la práctica } \\
\text { Debate sobre las conclusiones obtenidas }\end{array}$ \\
\hline
\end{tabular}

\section{Energía y temperatura}

El objetivo de esta práctica es observar el aumento de la temperatura de un gas al someterlo a una compresión tan rápida que no hubiera prácticamente transferencia de calor y pueda considerarse un proceso adiabático. De este modo, se verifica el comportamiento termodinámico de un sistema real.

Al comprimir un gas rápidamente, éste aprecia un aumento de su energía interna a causa del trabajo que se realiza contra el sistema. Con la compresión, aumenta la temperatura.

Los materiales necesarios para realizar la práctica son:

\section{- Placa Arduino}




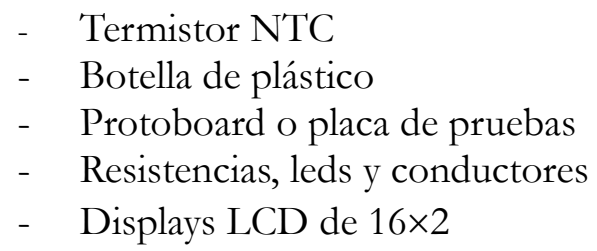

Se conectan los cables y los elementos según el esquema de la figura 1 , de tal manera que al someter a presión la botella de plástico, el termistor ubicado en su tapa detecta la variación de temperatura. Al variar la temperatura, el termistor varía su resistencia y, por lo tanto, la intensidad también se modifica.
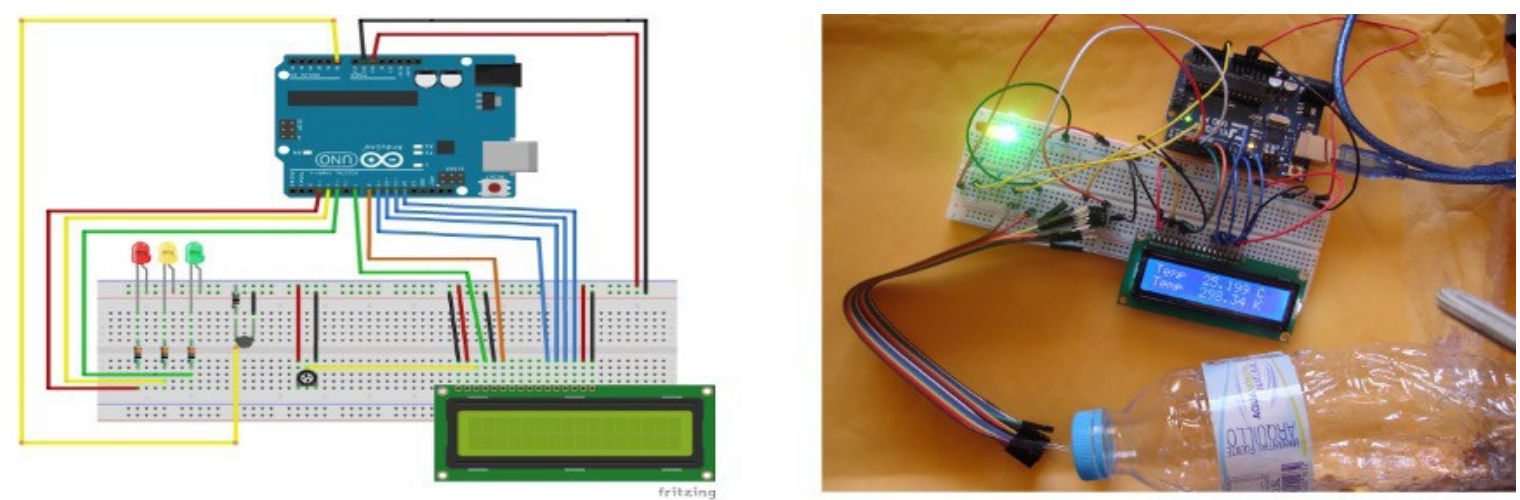

Figura 1. Esquema eléctrico del medidor de temperatura (elaborado con Fritzing) y fotografía del medidor de temperatura activado midiendo la temperatura ambiente (elaboración propia).

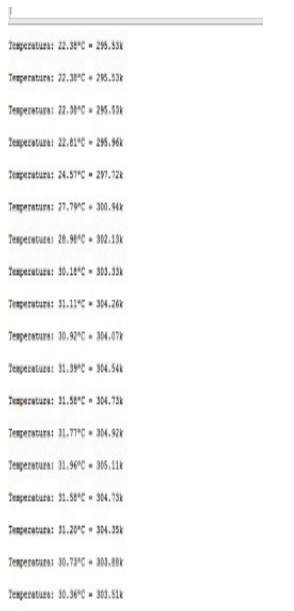

Figura 2. Toma de datos del medidor de temperatura al variar la presión (elaboración propia).
Estos valores son detectados por la placa de Arduino. El valor de la temperatura se muestra en el display tanto en grados centígrados como en grados Fahrenheit (figura 2), así el alumno relaciona las diferentes escalas de temperatura. Para que sea más vistoso, por medio de los leds se va indicando el rango de temperaturas. Dependiendo del color del led que se encienda, se encontrará en un rango de temperaturas u otro.

\section{Medir distancias}

El objetivo de esta práctica es comprender el funcionamiento de un sensor a través del sonido para medir la distancia a la que se encuentran los objetos. Esta técnica de ecolocación la utilizan animales como los murciélagos, ballenas y delfines para atrapar a sus presas.

Al emitir un pulso sónico corto, y determinar cuánto tiempo tarda en regresar el eco, se puede calcular la distancia a la que se encuentra el objeto en el que se refleja la señal, ya que se conoce la velocidad a la que se propaga el sonido. Para realizar la práctica se utiliza un sensor de ultrasonidos HC-SR04 (muy parecido a los sensores de aparcamiento de los coches modernos), el cual permite enviar estos pulsos ultrasónicos y recepcionar el eco de retorno. Al medir este tiempo, se obtiene la distancia hasta el obstáculo.

Los materiales necesarios para realizar la práctica son:

- Placa Arduino

- Sensor de ultrasonidos HC-SR04

- Protoboard o placa de pruebas

- Resistencias, leds y conductores

- Displays LCD de $16 \times 2$ 
Una vez conectada la placa de Arduino a una fuente de tensión, pila o al ordenador por el puerto USB, el sensor de ultrasonidos emite pulsos de alta frecuencia, no audibles por el ser humano. El sensor mide el tiempo entre el envío y la recepción de un pulso sonoro.

El esquema eléctrico se muestra en la figura 3 y en la figura 4 se muestra el circuito real.

La velocidad del sonido (en condiciones de temperatura $20^{\circ} \mathrm{C}$, al $50 \%$ de humedad y a presión atmosférica a nivel del mar) es de $343 \mathrm{~m} / \mathrm{s}$. Se transforman las unidades para calcular el tiempo que tarda el sonido en recorrer $1 \mathrm{~cm}$, dando

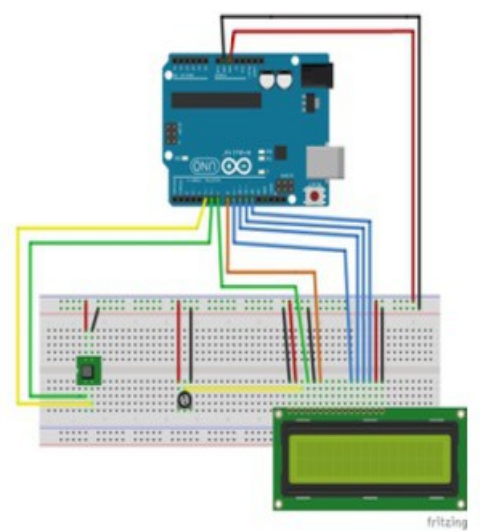
como resultado $343 \frac{\mathrm{m}}{\mathrm{s}} 100 \frac{\mathrm{cm}}{\mathrm{m}} \frac{1 \mathrm{~s}}{10^{6} \mu \mathrm{s}}=\frac{1}{29,2} \frac{\mathrm{cm}}{\mu \mathrm{s}}$.

Como resultado, el sonido tarda 29,2 microsegundos en recorrer un centímetro. Por tanto, se puede obtener la distancia en $\mathrm{cm}$, a partir del tiempo entre la emisión y recepción del pulso mediante la siguiente ecuación: $\operatorname{Distancia}(\mathrm{cm})=\frac{\text { Tiempo }(\mu \mathrm{s})}{29,2 \mu \mathrm{s} \times 2}$.

La distancia se ha dividido entre dos ya que el sensor mide el tiempo que tarda el pulso en ir y en volver.

Figura 3. Esquema eléctrico del medidor de distancias (elaborado con Fritzing).

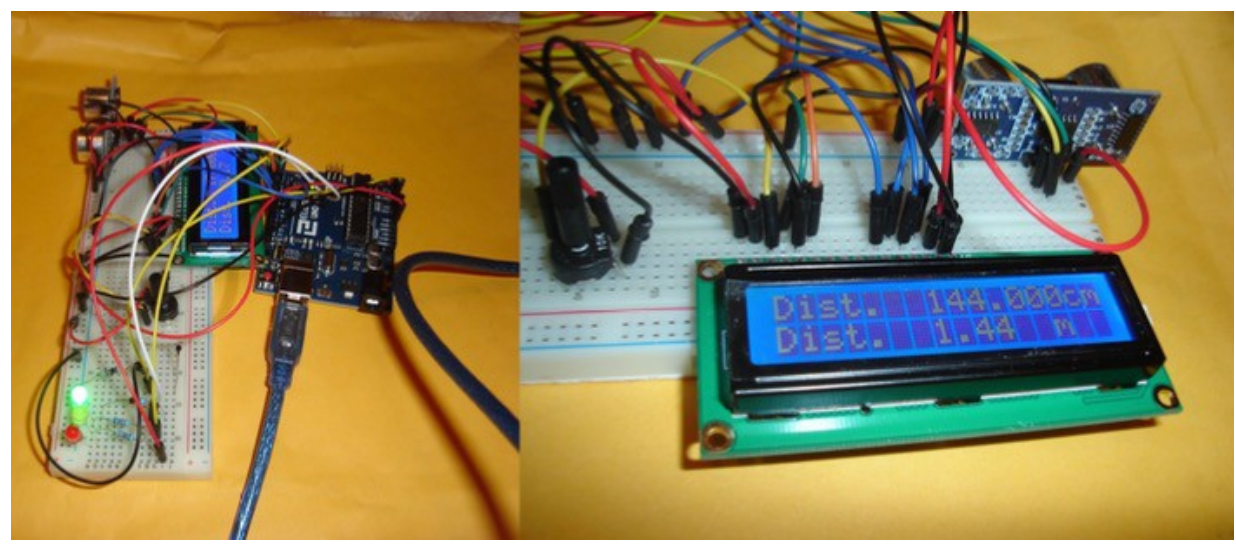

Figura 4. Fotografías del circuito medidor de distancias real en funcionamiento y detalle del display LCD donde se muestra la distancia medida a un objeto en centímetros y en metros.

\section{Mando a distancia}

El objetivo de esta práctica es conocer los rayos infrarrojos, las ondas electromagnéticas y su funcionamiento en los mandos a distancia.

Las ondas electromagnéticas se caracterizan, principalmente por su frecuencia o por su inversa, que es la longitud de onda multiplicada por la velocidad de propagación de la onda. Un mando a distancia de infrarrojos funciona emitiendo pulsos de luz infrarroja (de mayor longitud de onda que la luz visible).

Las ventajas que posee la luz infrarroja para realizar mandos a distancia son las siguientes:

- Utilizan luz en una frecuencia menor que la luz visible.

- La luz del mando no molesta en la oscuridad.

- Tiene relativamente poco alcance, pero no se suele ubicar el dispositivo de reproducción más allá de 2 o 3 metros de distancia. 
Aunque también posee algunos inconvenientes. El principal es que hay múltiples elementos que emiten radiación infrarroja, como las personas, los animales, los radiadores o el Sol; la cual puede interferir en el mando a distancia. La solución es lanzar un tren de ondas estable con la información que se quiere enviar, es decir, modular la onda que se envía. El mensaje tiene que seguir unas determinadas normas (forma de los pulsos, duración y contenido) que deben ser reconocidas tanto por el emisor como por el receptor para que la comunicación sea correcta.

Los materiales necesarios para realizar la práctica son:

- Placa Arduino

- Receptor de infrarrojos AX1838HS

- Protoboard o placa de pruebas

- Resistencias, leds y conductores

- Buzzer

El esquema de montaje de la práctica se muestra en la figura 5.
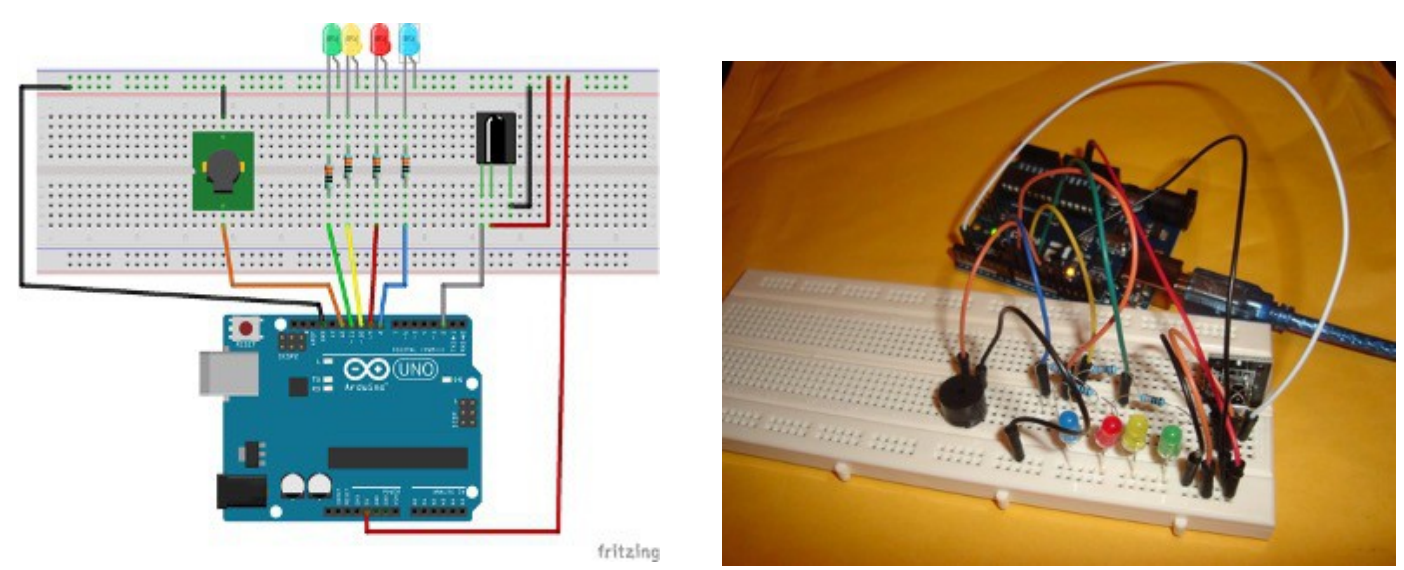

Figura 5. Esquema eléctrico del receptor de infrarrojos y los elementos que se van a accionar (elaborado con Fritzing) y fotografía del circuito receptor del mando a distancia donde se aplican las acciones del mando a distancia (elaboración propia).

En primer lugar, hay que conocer el código asignado a cada uno de los botones del mando a distancia (el código en el cual envía el emisor las señales). Tras conocer el código, se le concede a cada botón una acción a realizar. Estas acciones se recogen en la tabla 2.

Tabla 2. Acciones asignadas a cada botón del mando a distancia.

\begin{tabular}{|c|c|}
\hline Botón del mando a distancia & Acción a realizar \\
\hline 1 & Se enciende un led azul \\
\hline 2 & Se enciende un led rojo \\
\hline 3 & Se enciende un led amarillo \\
\hline 4 & Se enciende un led verde \\
\hline 5 & Emite el buzzer la canción de "Star Wars" \\
\hline On-Off & Apagado de los leds \\
\hline Botón de avanzar más rápido & Luces modo "coche fantástico" \\
\hline
\end{tabular}

\section{Temperatura y humedad}

En esta práctica el principal objetivo es formular la hipótesis de trabajo para la resolución de un problema real que sea el elemento detonador del proceso de enseñanza-aprendizaje. 
Se parte de una situación ficticia pero coherente con la realidad: Un vecino que posee un almacén de frutas y verduras comenta lo que le ha ocurrido la semana pasada para ver si se le puede ayudar en su problema. La fruta y verdura que almacena se ha echado a perder y, por lo tanto, ha tenido pérdidas. Si siempre la almacena en el mismo sitio, ¿por qué le ha pasado esto? La puntualización que nos aporta es que esa semana ha babido una ola de calor y el tiempo ba sido muy seco.

Se intenta de este modo proponer un reto a los alumnos, como factor motivacional, para mejorar la enseñanza de las ciencias (Marra, Jonassen, Palmer y Luft 2014), para que el estudiante vea la posibilidad de desarrollo profesional y la aplicación de lo que aprende en su vida cotidiana (Kilroy 2004).

Una vez planteado el problema, se realiza una lluvia de ideas de la principal causa, formulando entre todos la hipótesis de trabajo: aparte de la temperatura y del tiempo, que juegan un papel muy importante de forma conjunta, hay otros factores destacados, como la humedad, la cual si está por debajo de la recomendada, favorece la desecación y pérdida de peso excesiva en las frutas y verduras, y si está por encima, el riesgo de contaminación por moho aumenta. El esquema de montaje para la realizar la práctica y el montaje real se muestran en la figura 6 . Se propone el uso del sensor DHT11 a utilizar en la placa de Arduino para monitorizar tanto la humedad relativa como la temperatura (figura 7).

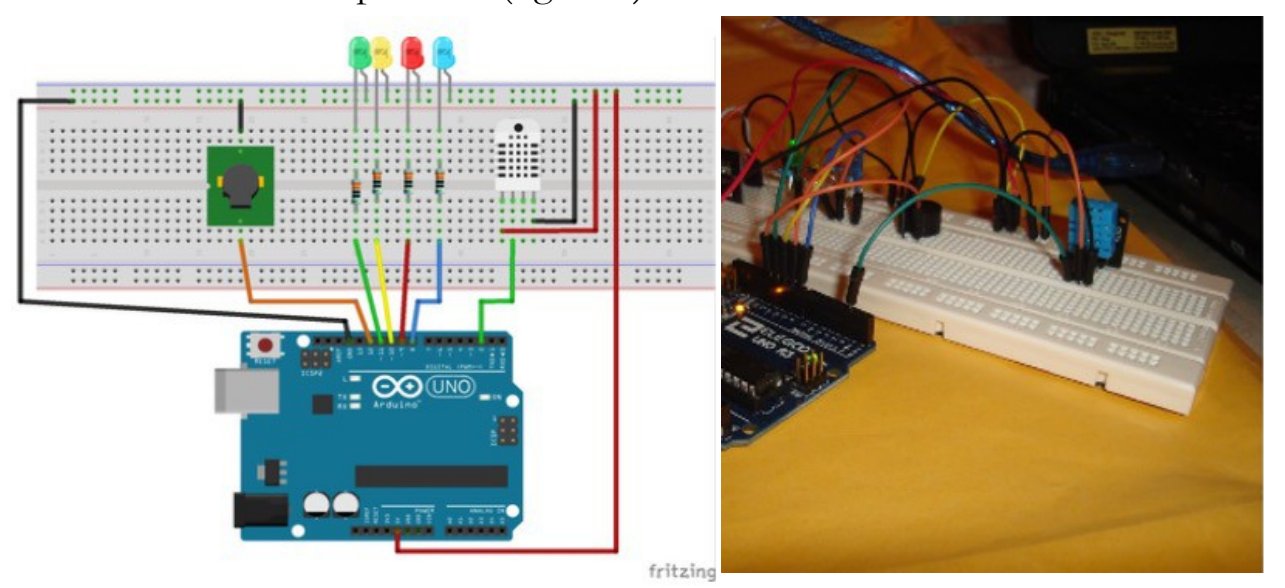

Figura 6. Esquema eléctrico (elaborado con Fritzing) y fotografía del montaje del circuito medidor de las variables temperatura y humedad (elaboración propia).

Los materiales necesarios para realizar la práctica son:

- Placa Arduino

- Sensor DHT11

- Protoboard o placa de pruebas

- Resistencias, leds (verde, azul, amarillo y rojo) y conductores

- Buzzer

Una vez conocidos los parámetros a los cuales debe estar el ambiente para que se conserve mejor la fruta y la verdura, es decir, la humedad y la temperatura del espacio de almacenaje, se codifica en el código de Arduino. Si la temperatura y la humedad están en los valores correctos, se encenderá el led verde. Si la temperatura se mantiene en el nivel, pero la humedad no se encuentra dentro del rango de conservación de los alimentos se iluminará el led azul. Si, por el contrario, la humedad se encuentra en el rango adecuado pero la temperatura no, se encenderá el led amarillo. Por último, si ambos parámetros están fuera de los rangos óptimos, se iluminará el led rojo y, además, sonará una sirena para advertir tanto acústica como visualmente. 


\section{Vúmetro}

El objetivo de esta práctica es comprender el efecto que produce el nivel de presión sonora en la salud auditiva al escuchar música con auriculares. Para que los alumnos analicen si supone un riesgo escuchar la radio a través de los auriculares, se da a los estudiantes una tabla con información de la relación entre el tiempo de exposición y el nivel de presión sonora (tabla 3). Aunque también podría ser información sobre la que los estudiantes indagaran previamente.

Los alumnos realizan un vúmetro, dispositivo que indica el nivel de volumen en un aparato de audio. Por medio de la tabla 3 tienen que ejercitar el diseño de un vúmetro con ocho leds, que indicarán el nivel de presión sonora. Los tres primeros niveles se indican con leds de color verde, los tres siguientes con leds amarillos y, el resto, con leds rojos.

Tabla 3. Relación entre el nivel de presión sonora y el tiempo de exposición máximo sin peligro (Caamaño 2011).

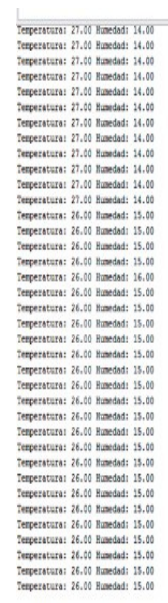

Figura 7. Toma de datos del medidor de temperatura y humedad (elaboración propia).

\begin{tabular}{|c|c|}
\hline $\begin{array}{c}\text { Nivel de presión sonora } \\
\text { (dB) }\end{array}$ & $\begin{array}{c}\text { Tiempo de exposición } \\
\text { (h) }\end{array}$ \\
\hline 80 & 16 \\
\hline 85 & 8 \\
\hline 90 & 4 \\
\hline 95 & 2 \\
\hline 100 & 1 \\
\hline 105 & $1 / 2$ \\
\hline 110 & $1 / 4$ \\
\hline 115 & $1 / 8$ \\
\hline
\end{tabular}

$\mathrm{Al}$ aumentar el volumen aumenta el nivel de presión sonora. Cuando mayor sea el volumen, más leds se encenderán. Los alumnos deben comprobar el volumen con el que escuchan música y analizar si es dañino para la salud.

Los materiales necesarios para realizar la práctica son:

- Placa Arduino

- 8 leds (3 verdes, 3 amarillos y 2 rojos)

- Protoboard o placa de pruebas

- Resistencias y conductores

- Cable de audio

- Una fuente de audio

El esquema de montaje para la realizar la práctica y el montaje real se muestran en la figura 8. 

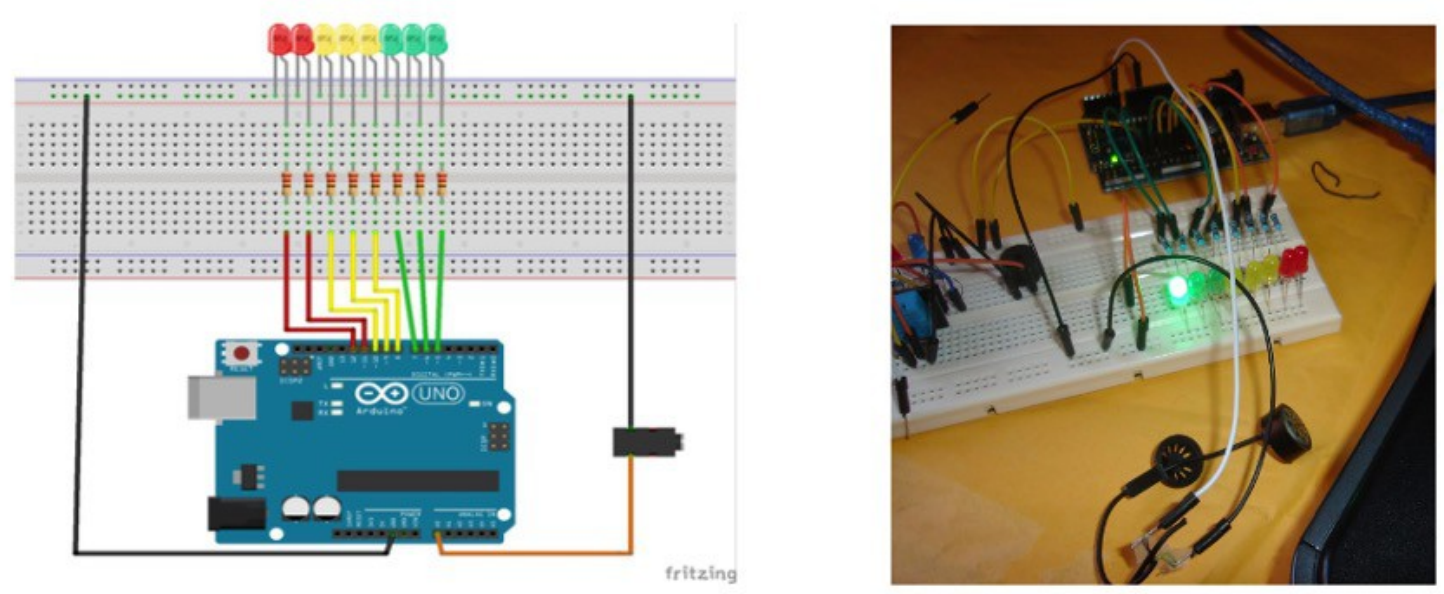

Figura 8. Esquema eléctrico del vúmetro (elaborado con Fritzing) y montaje real (elaboración propia).

La señal de audio es introducida a la placa de Arduino por una entrada analógica. Esta entrada analógica se va comparando con los valores máximos que cada led tiene asignados, para ello, se ha repartido el voltaje máximo de entrada entre los ocho leds. Si el valor leído es mayor que el valor que se le concede a cada intervalo de voltaje de los leds, se encenderán los leds que estén por debajo de este valor.

En la figura 9 se distinguen los valores que va leyendo el software de Arduino y ordena que se encienda el led correspondiente. Al finalizar el montaje, los alumnos prueban el nivel de volumen con el que ellos mismos escuchan la música a través de los auriculares. Tienen que realizar una medida del promedio de nivel de presión sonora que se alcanza mientras están escuchando música. Como se ha comentado anteriormente, si el volumen está muy alto se encienden los leds rojos, mientras que si el nivel del volumen es moderado o bajo, únicamente se encienden los leds verdes y/o los amarillos. Una vez obtenido el promedio estiman las horas semanales que escuchan la música a este nivel y calculan el tiempo al que están expuestos diariamente. Para concluir, se realiza un debate con los datos obtenidos en la actividad y se analiza si está en peligro la salud

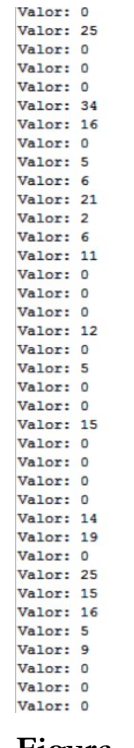
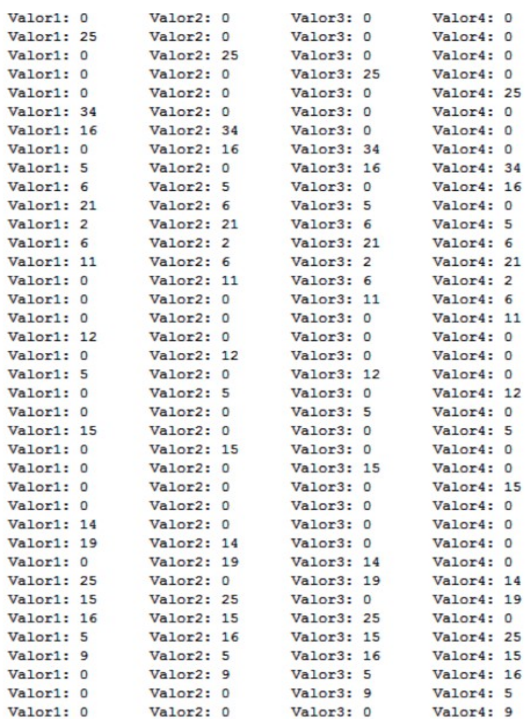

Figura 9. Toma (elaboración propia) auditiva de las personas.

\section{$\mathrm{pH}$ y notas musicales}

El objetivo de esta práctica es conocer la relación entre el $\mathrm{pH}$ y la conductividad eléctrica de ciertas frutas y así generar notas musicales a través de la placa de Arduino, es decir, realizar un piano con las frutas dependiendo de su acidez-conductividad.

$\mathrm{Al}$ investigar sobre la caracterización físico-química de ciertas frutas se parte de la hipótesis de que a mayor grado de acidez de un cítrico va a tener una mayor conductividad eléctrica, dado que cuanto más ácido sea el cítrico, éste actúa como un electrolito más fuerte y, en algunos casos, el contenido ácido es lo suficientemente alto como para crear un voltaje que puede alimentar pequeños componentes electrónicos. 
El $\mathrm{pH}$ mide la concentración de iones de hidrógeno $\mathrm{pH}=-\log \left[\mathrm{H}^{+}\right]$. Al poseer mayor cantidad de iones, la conductividad aumenta. La conductividad nos indica si el material permite el paso de los electrones, es decir, si no opone resistencia a que pasen los electrones a través de él.

Se han elegido las frutas ácidas para observar su conductividad. Para ello, se realiza la medida de su pH y su voltaje (véase la tabla 4) observando que hay una relación inversamente proporcional, al ser la fruta más ácida, con más $\mathrm{pH}$, disminuye más el voltaje por lo que se favorece la circulación de electrones y su conductividad es mayor.

Los materiales necesarios para realizar la práctica son:

- Placa Arduino

Tabla 4. Relación entre el pH y el

voltaje de las frutas.

\begin{tabular}{|r|c|c|}
\hline & $\mathbf{p H}$ & Voltaje $(\mathbf{V})$ \\
\hline Lima & 2 & 1,08 \\
\hline Limón & 2,2 & 0,72 \\
\hline Manzana & 3 & 0,66 \\
\hline Pomelo & 3,3 & 1,11 \\
\hline Naranja & 3,6 & 1,09 \\
\hline Kiwi & 5,8 & 0,20 \\
\hline
\end{tabular}

- Protoboard o placa de pruebas

- Resistencias y conductores

- Frutas (lima, limón, manzana, pomelo, naranja y kiwi)

- Buzzer

El esquema de montaje para realizar la práctica y el montaje real se pueden ver en la figura 10. Las figuras 11 y 12 muestran la toma de datos de la práctica.
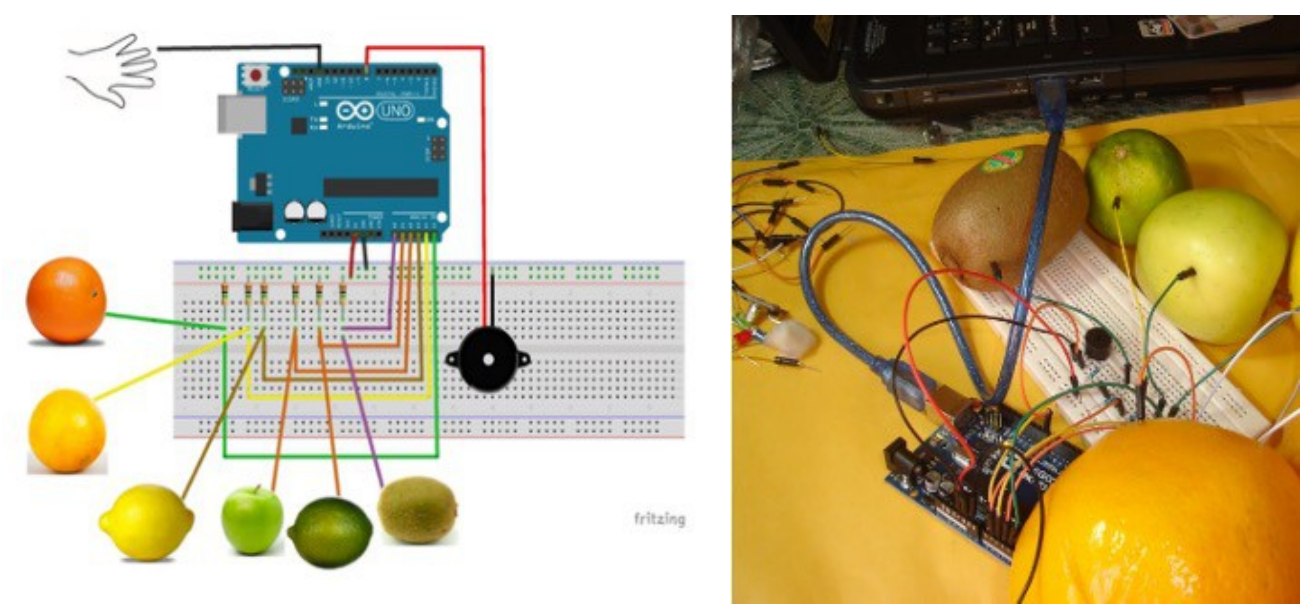

Figura 10. Esquema eléctrico del piano (elaborado con Fritzing) y montaje real del piano con frutas (elaboración propia).

El divisor de tensión, que está formado por una resistencia de $1 \mathrm{M} \Omega$ y la resistencia propia de cada fruta, es alimentado a través de la placa de Arduino. El voltaje obtenido a la salida del divisor de tensión es enviado a una entrada analógica de Arduino. Al cerrar el circuito, se produce una diferencia de potencial en los bornes de las resistencias obteniendo de esta manera una tensión menor a los $5 \mathrm{~V}$ iniciales en función de la resistencia que oponga cada fruta debido a su acidez. Dependiendo de este nivel de tensión, Arduino envía la señal al buzzer produciendo una nota diferente en cada fruta.

\section{Evaluación}

Los alumnos deben elaborar una memoria de prácticas en la cual mostrarán los resultados a los que han llegado y las conclusiones y reflexiones obtenidas a partir de cada práctica. 


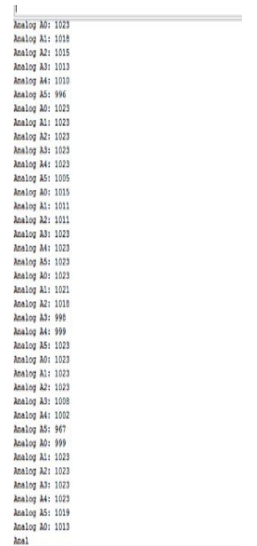

Figura 11. Toma de datos de las entradas analógicas de Arduino sin cerrar el circuito (elaboración propia).
Para evaluar el desempeño de cada equipo de estudiantes al realizar las prácticas se ha elaborado una posible rúbrica (véase la tabla 5) en la que se han puntuado los indicadores de 1 a 4. Para su confección se ha tenido en cuenta las competencias que se trabajarán con el conjunto de las prácticas.

Se ha elegido la rúbrica debido a que constituye una herramienta de evaluación no convencional que puede definirse como una guía para evaluar la calidad de los trabajos y el nivel de ejecución alcanzado por los estudiantes en una amplia variedad de tareas complejas, especificando los criterios a considerar y los niveles de adecuación en cada uno de ellos. Además, después de completar la memoria de prácticas se pueden evaluar otras habilidades como: la comunicación escrita, la capacidad de usar aplicaciones informáticas, razonamiento crítico y la gestión de la información.

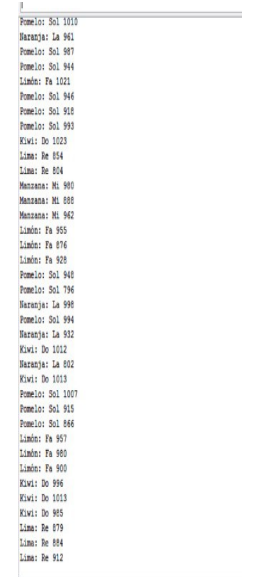

Figura 12. Toma de datos del piano con frutas (elaborael ción propia).

Tabla 5. Posible rúbrica para la evaluación de las prácticas de cada grupo.

\begin{tabular}{|c|c|c|c|c|}
\hline Indicadores & $\begin{array}{l}\text { Nivel } 1 \\
\text { (suspenso) }\end{array}$ & $\begin{array}{l}\text { Nivel } 2 \\
\text { (aprobado) }\end{array}$ & $\begin{array}{l}\text { Nivel } 3 \\
\text { (notable) }\end{array}$ & $\begin{array}{l}\text { Nivel } 4 \\
\text { (sobresaliente) }\end{array}$ \\
\hline $\begin{array}{l}\text { Trabajo en equipo } \\
20 \%\end{array}$ & $\begin{array}{l}\text { Los integrantes del } \\
\text { grupo han trabajado } \\
\text { por separado. }\end{array}$ & $\begin{array}{l}\text { Varios integrantes no } \\
\text { trabajan adecuadamente. }\end{array}$ & $\begin{array}{l}\text { Algún integrante del } \\
\text { grupo no trabaja } \\
\text { adecuadamente. }\end{array}$ & $\begin{array}{l}\text { Notorio el trabajo en } \\
\text { equipo realizado por todos } \\
\text { los integrantes. }\end{array}$ \\
\hline Organigrama $2 \%$ & Inexistente & $\begin{array}{l}\text { Incompleto y difícil de } \\
\text { entender }\end{array}$ & $\begin{array}{l}\text { Se entiende el diseño del } \\
\text { programa }\end{array}$ & $\begin{array}{l}\text { Organigrama claro para } \\
\text { entender el diseño del } \\
\text { programa. }\end{array}$ \\
\hline $\begin{array}{l}\text { Uso de instruc- } \\
\text { ciones y algorit- } \\
\text { mos. } \\
\text { Funcionamiento } 20 \\
\%\end{array}$ & No funciona. & Tiene fallos importantes. & $\begin{array}{l}\text { No es completo, aunque } \\
\text { tiene fallos sin } \\
\text { importancia. }\end{array}$ & Adecuado y completo. \\
\hline $\begin{array}{l}\text { Conceptos } \\
\text { científicos } 20 \%\end{array}$ & Comete errores graves & $\begin{array}{l}\text { Presenta alguna } \\
\text { deficiencia el desarrollo. }\end{array}$ & $\begin{array}{l}\text { Conoce los contenidos } \\
\text { y/o comete algún error en } \\
\text { el desarrollo. }\end{array}$ & $\begin{array}{l}\text { Domina los contenidos, y } \\
\text { plantea correctamente el } \\
\text { caso de estudio. }\end{array}$ \\
\hline $\begin{array}{l}\text { Contenido y } \\
\text { organización. } \\
\text { Relación Textos / } \\
\text { Gráficos 9\% } \\
\end{array}$ & $\begin{array}{l}\text { Mínimo. } \\
\text { No está clara o no es } \\
\text { lógica. }\end{array}$ & $\begin{array}{l}\text { Información básica sobre } \\
\text { el tema. }\end{array}$ & $\begin{array}{l}\text { Conocimiento básico, } \\
\text { buen contenido y } \\
\text { organización lógica }\end{array}$ & $\begin{array}{l}\text { Conocimiento del tema es } \\
\text { excelente, contenido bien } \\
\text { organizado }\end{array}$ \\
\hline $\begin{array}{l}\text { Análisis de } \\
\text { resultados } 15 \%\end{array}$ & $\begin{array}{l}\text { Formato incorrecto } \\
\text { y/o no los interpreta } \\
\text { correctamente. }\end{array}$ & $\begin{array}{l}\text { Da algún resultado sin el } \\
\text { formato requerido } \\
\text { (unidades, precisión, } \\
\text { error...) y/o con } \\
\text { bastantes deficiencia en la } \\
\text { interpretación de los } \\
\text { mismos. }\end{array}$ & $\begin{array}{l}\text { Da los resultados con el } \\
\text { formato requerido } \\
\text { (unidades, precisión, } \\
\text { error...) pero con alguna } \\
\text { deficiencia en la } \\
\text { interpretación de los } \\
\text { mismos. }\end{array}$ & $\begin{array}{l}\text { Da los resultados con el } \\
\text { formato requerido } \\
\text { (unidades, precisión, } \\
\text { error...) y los interpreta } \\
\text { correctamente }\end{array}$ \\
\hline
\end{tabular}




\section{Conclusiones}

En base a la búsqueda, recopilación y comprobación de experiencias en este trabajo se logra recopilar un compendio de prácticas para estudiantes de Secundaria y/o Bachillerato con el acceso a la plataforma Arduino y su comunidad de código abierto.

Actualmente, nos encontramos en una sociedad globalizada y cambiante, donde las generaciones nacidas en el nuevo siglo han convivido desde siempre con las tecnologías de la información y la comunicación (TIC). Parece evidente que la educación debe dar respuesta a las necesidades de la sociedad, aunque como indican diversos autores, aún no se está consiguiendo formar a estudiantes para la sociedad del siglo XXI, ya que aspectos como la multiculturalidad, la digitalización de la información y la importancia de las redes sociales no se abordan con suficiente importancia en nuestras aulas (Gutiérrez-Porlán, Román-García y Sánchez-Vera 2018). Resulta entonces inminente realizar cambios metodológicos en los distintos espacios curriculares que conforman la educación obligatoria, a fin de favorecer no solo los respectivos aprendizajes disciplinares, sino también el desarrollo de esas competencias digitales.

El uso de las estrategias de aprendizaje activo, incluidas las actividades prácticas y el trabajo de laboratorio, han demostrado ser beneficiosas para la comprensión y el éxito de los estudiantes en las aulas de ciencias (Rywalt, Varney y Mutton 2019). Para ello, la utilización de las TIC aumenta la motivación de los alumnos, ya que el aprendizaje les resulta más atractivo y divertido, lo que hace que los estudiantes dediquen más tiempo al estudio y se encuentren más implicados en todas las actividades (Ferro Soto, Martínez Senra y Otero Neira 2009).

En este ámbito, los sistemas de adquisición de datos juegan un papel fundamental en todos los ámbitos de la ciencia y de la tecnología. Como se ha visto en el marco teórico, el uso de los sensores de captación de datos, con Arduino, se ha extendido en el ámbito educativo aportando metodologías activas por medio de proyectos y prácticas en los laboratorios. Los estudios muestran que Arduino es un instrumento idóneo tanto para la etapa de Secundaria como para la de Bachillerato porque es flexible, de precio asequible y se adapta a diferentes prácticas y aplicaciones dependiendo de cómo se programe y los sensores que se utilicen.

Una de las motivaciones de este trabajo era hacer que las prácticas fueran lo más económicas posible; esto permitiría a algunas escuelas secundarias que no pueden pagar el equipo de laboratorio tradicional implementar estas prácticas en sus centros y proporcionar los beneficios del aprendizaje activo a sus estudiantes.

La batería de actividades que se presenta en este trabajo enseña cómo por medio de la tecnología se pueden mostrar resultados científicos y llevarlos a la práctica en las asignaturas de Física y Química de los centros escolares, tanto en los laboratorios o en la propia aula y adaptándolas a las diversas edades de los estudiantes y su nivel académico.

Durante la realización de las prácticas, los alumnos trabajarán en grupos para que exista trabajo colaborativo, deben plantear los circuitos para solucionar el problema de partida bajo la supervisión y ayuda del docente y, finalmente, redactar los datos obtenidos y las conclusiones a las que se ha llegado. Los experimentos se diseñan para llevarlos a cabo por medio de Arduino, adquiriendo los datos a través de los sensores y controlándolos para obtener los resultados.

Los estudios revisados muestran cómo la utilización de la tecnología de sensores integrada con Arduino puede ayudar a los estudiantes a entender cambios físicos y químicos, relación entre variables dependientes y obtención de datos en tiempo real con el empleo de recursos 
sencillos. Consiguiendo favorecer la tan ansiada alfabetización científica y digital, objetivos que hoy día persigue la enseñanza obligatoria (Bravo, Bouciguez y Braunmüller 2019).

Por tanto, con el uso de esta herramienta se espera potenciar la comunicación e interacción entre el alumnado y, facilitar la comprensión de los conceptos científicos. Además, de fomentar las habilidades digitales y técnicas, por trabajar con el ordenador y realizar montajes de circuitos eléctricos; así como la creatividad y el pensamiento crítico por medio de la tecnología.

\section{Referencias}

Alegre Buj M. S. (2020) Trabajos prácticos de Física y Química con Arduino. Mendeley Data, v2.

Area M., Cepeda O., Romero L. (2018) El uso escolar de las TIC desde la visión del alumnado de Educación Primaria, Secundaria y Bachillerato. Educatio Siglo XXI 36(2), 229-254.

Aufschnaiter C. V., Aufschnaiter S. V. (2007) University students activities, thinking and learning during laboratory work. European Journal of Physics 28(3), 50-60.

Blanco López Á., Prieto Ruz T. (2004) Un esquema para investigar el progreso en la comprensión de los alumnos sobre la naturaleza de la materia. Revista de Educación 335, 445-465.

BOE (2015) Orden ECD/65/2015, de 21 de enero, por la que se describen las relaciones entre las competencias, los contenidos y los criterios de evaluación de la educación primaria, la educación secundaria obligatoria y bachillerato. $\mathrm{BOE} \mathrm{n}^{\circ} 25$, de 29 de enero de 2015.

Bravo B., Bouciguez M. J., Braunmüller. M. (2019) Una propuesta didáctica diseñada para favorecer el aprendizaje de la Inducción Electromagnética básica y el desarrollo de competencias digitales. Revista Eureka sobre Enseñanza y Divulgación de las Ciencias 16 (1), 1203.

Caamaño A. (2011) Física y química: investigación, innovación y buenas prácticas. Barcelona: Graó.

Carranza P., Gianna V., Gómez M., Larrosa N., López A., Marín M., Martínez M., Martínez Riachi S., Melchiorre M., Peci M., Ribotta P., Saldis Heredia, N., Severini H., Vaca Chávez J., Yorio D. (2013) Sensores. Una exitosa experiencia interdisciplinar en la enseñanza de las ciencias. Córdoba: Editorial Brujas y Facultad de Ciencias Exactas, Físicas y Naturales de la UNC.

Ferro Soto C., Martínez Senra A., Otero Neira M. C. (2009) Ventajas del uso de las TIC en el proceso de enseñanza-aprendizaje desde la óptica de los docentes universitarios españoles. Revista Electrónica de Tecnología Educativa 29, 1-12.

Garrigós A., Marroqui D., Blanes J. M., Gutierrez R., Blanquer I., Cantó M. (2017) Designing Arduino electronic shields: Experiences from secondary and university courses. pp. 934-937 en IEEE Global Engineering Education Conference (EDUCON 2017).

Gutiérrez-Porlán I., Román-García M., Sánchez-Vera M. (2018) Estrategias para la comunicación y el trabajo colaborativo en red de los estudiantes universitarios. Comunicar 54 (26), 91-100.

Instituto Nacional de Tecnologías Educativas y Formación del Profesorado (INTEF) (2017) Marco Común de Competencia Digital Docente. Madrid: Ministerio de Educación, Cultura y Deporte.

Jamieson P. (2011) Arduino for teaching embedded systems. are computer scientists and engineering educators missing the boat? p. 1 en Proceedings of the International Conference 
on Frontiers in Education: Computer Science and Computer Engineering (FECS). The Steering Committee of The World Congress in Computer Science, Computer Engineering and Applied Computing (WorldComp).

Kilroy D. A. (2004) Problem based learning. Emergency Medicine Journal: EMJ 21(4), 411-413.

Kinchin J. (2018) Using an Arduino in physics teaching for beginners. Physics Education 53 (6), 063007.

LOMCE (2014) Ley Orgánica 8/2013, de 9 de diciembre, para la mejora de la calidad educativa. BOE $\mathrm{n}^{\mathrm{o}} 295$, de 10 de diciembre de 2013, 97858-97921.

Marra R. M., Jonassen D. H., Palmer B., Luft S. (2014) Why Problem-Based Learning Works: Theoretical Foundations. Journal on Excellence in College Teaching 25 (3-4), 221-238.

Moya A. A. (2018) An Arduino experiment to study free fall at schools. Physics Education 53 (5), 055020 .

Oliveira P. M., Hedengren J. D. (2019) An APMonitor Temperature Lab PID Control Experiment for Undergraduate Students. pp. 790-797 en 24th IEEE International Conference on Emerging Technologies and Factory Automation (ETFA), Zaragoza.

Parreira P., Yao E. (2018) Experimental design laboratories in introductory physics courses: enhancing cognitive tasks and deep conceptual learning. Physics Education 53 (5), 055012.

Pérez Carmona M. C., Esper L. B. (2005) Algunos problemas en la conceptualización de ondas mecánicas. Enseñanza de las Ciencias, número extra, VII Congreso, 1-6.

Pisa (2018) Programa para la Evaluación Internacional de los Estudiantes. Informe español. Madrid: Ministerio de Educación y Formación Profesional.

Pozo J. I., Gómez M. A. (2009) Aprender y enseñar ciencia: del conocimiento cotidiano al conocimiento cientifico. Madrid: Ediciones Morata.

Prima E. C., Karim S., Utari S., Ramdani R., Putri E. R. R., Darmawati S. M. (2017) Heat Transfer Lab Kit using Temperature Sensor based Arduino ${ }^{\mathrm{TM}}$ for Educational Purpose. Procedia Engineering 170, 536-540.

Rodríguez-Sánchez M. C., Torrado-Carvajal A., Vaquero J., Borromeo S., HernandezTamames J. A. (2016) An embedded systems course for engineering students using open-source platforms in wireless scenarios. IEEE Transactions on Education 59 (4), 248254.

Rywalt C. E., Varney M. E., Mutton Z. M. (2019) Physics Education Research on Inexpensive ActiveLearning Lab Modules. Massachusetts: Worcester Polytechnic Institute. 